\title{
Investigation of the Effect of Electrode Geometry on Spark Ignition
}

\author{
S.P.M. Bane ${ }^{\mathrm{a}, *}$, J.L. Ziegler ${ }^{\mathrm{b}}$, J.E. Shepherd ${ }^{\mathrm{c}}$ \\ ${ }^{a}$ School of Aeronautics and Astronautics, Purdue University \\ 701 W. Stadium Ave. \\ West Lafayette, Indiana 47907, USA \\ ${ }^{b}$ National Bioenergy Center, National Renewable Energy Laboratory \\ 15013 Denver W Pkwy, MS 3311, Golden, CO 80401 \\ ${ }^{c}$ Graduate Aerospace Laboratories, California Institute of Technology \\ 1200 E. California Blvd., MC 205-45 \\ Pasadena, California 91125, USA
}

\begin{abstract}
High-speed schlieren visualization and numerical simulations are used to study the fluid mechanics following a spark discharge and the effect on the ignition process in a hydrogen-air mixture. A two-dimensional axisymmetric model of spark discharge in air and spark ignition was developed using the non-reactive and reactive Navier-Stokes equations including mass and heat diffusion. The numerical method employs structured adaptive mesh refinement software to produce highly-resolved simulations, which is critical for accurate resolution of all the physical scales of the complex fluid mechanics and chemistry. The simulations were performed with three different electrode geometries to investigate the effect of the geometry on the fluid mechanics of the evolving spark kernel and on flame formation. The computational results were compared with high-speed schlieren visualization of spark and ignition kernels. It was shown that the spark channel emits a blast wave that is spherical near the electrode surfaces and cylindrical near the center of the spark gap, and thus is highly influenced by the electrode geometry. The ensuing competition between spherical and cylindrical expansion in the spark
\end{abstract}

\footnotetext{
${ }^{\text {*N }}$ Full-length article submitted to Combustion and Flame.

*Corresponding author: sbane@purdue.edu

ph: +1 (765) 494-9364

fax: $+1(765)$ 494-0307
} 
gap and the boundary layer on the electrode surface both generate vorticity, resulting in the toroidal shape of the hot gas kernel and enhanced mixing. The temperature and rate of cooling of the hot kernel and mixing region are significantly effected by the electrode geometry and will have a critical impact on ignition. In the flanged electrode configuration the viscous effects generate a multidimensional flow field and lead to a curved flame front, a result not seen in previous work. Also, the high level of confinement by the flanges results in higher gas temperatures, suggesting that a lower ignition energy would be required. The results of this work provide new insights on the roles of the various physical phenomena in spark kernel formation and ignition, in particular the important effects of viscosity, pressure gradients, electrode geometry, and hot gas confinement.

Keywords: spark ignition, flames, electrode

\section{Introduction}

Determining the risk of accidental ignition of flammable mixtures is an important element in industrial and aviation safety. Traditionally, the method for studying ignition hazards of fuels is to determine the minimum ignition energy (MIE) through testing using a capacitive spark discharge as the ignition source. There have been extensive experimental studies to determine the minimum ignition energies of various flammable mixtures. However, recent investigations [1-3] of spark ignition suggest that ignition is statistical in nature rather than characterized by a single threshold value as presented in classic MIE data. Minimum ignition energy is found to depend strongly on the specifics of the geometry and the electrical discharge, complicating experimental investigations. There is minimal discussion in the literature on the effect on ignition of the spark channel geometry resulting from the electrical discharge and electromagnetic effects. In previous work [3], it was found that these effects contribute to variability of the ignition test results near the traditional MIE. Developing numerical tools to reliably predict ignition in different geometries is one of the outstanding issues in combustion science.

Much of the previous work on simulating ignition has idealized the problem and treated one-dimensional spherical and cylindrical spark kernels. Several authors have used two-dimensional simulations of spark discharge in a non-reactive gas [4-7] to investigate the fluid mechanics involved in the spark 
ignition process, and two-dimensional simulations of ignition are discussed in [8-16]. In all the two-dimensional studies, the classic toroidal shape of the hot gas kernel is observed, which occurs due to fluid flow inward toward the gap center. In most of these studies only one electrode geometry is considered and the simulations are not sufficiently resolved to capture all aspects of the fluid motion. Akram [5] and Thiele et al. [10] performed simulations for several electrode geometries, however, the geometries were limited to blunt and cone-shaped electrodes with diameters of 1 to $2 \mathrm{~mm}$. Most recently, Han et al. simulated ignition in methane-air [14] and hydrogen-air [15] mixtures and studied the effects of temperature, electrode gap distance, electrode size (cylindrical electrodes), and spark duration on the predicted minimum ignition energy (MIE). These studies used detailed chemistry and provided new insight on the effect of different spark parameters on ignition. However, the simulations did not appear to resolve the details of the fluid mechanics, and the authors primarily considered the temperature rise in the flame kernel and the heat loss to the electrodes. Also, many of the predicted MIE values were very large compared to historical values, suggesting that the model is still not complete. Nakaya et al. [16] also performed simulations of spark ignition in methane-air mixtures and focused on the interaction of chemical reactions, flow fields, and diffusion processes during ignition. The ability to accurately resolve these different physical processes is critical for a successful model and essential to our understanding of the ignition process. While the work of Nakaya et al. provides significant advancement towards accurate model development, the simulations were limited by low grid resolution and a single electrode geometry.

Many investigators have also performed experimental studies on visualizing spark discharge and ignition using optical and laser techniques. Experiments have been done to visualize the fluid mechanics of the evolving spark and ignition kernels using shadowgraph and schlieren visualization [4, 17-25] and interferometry [4, 26]. Laser diagnostics, such as laser-induced fluorescence (LIF) $[12,23,27]$ and spectroscopy [23, 27], have also been implemented to measure characteristics of the spark kernel such as temperature and magnitude of $\mathrm{OH}$ radicals. In all of these studies, the electrode geometry is not varied, and there is no direct comparison with two-dimensional simulations.

The plasma chemistry as well as the details of the spark channel formation will also largely effect the ignition process. Significant advances have been made in understanding the plasma chemistry and electrodynamics of shortduration electrical discharges. Most of the recent work has focused on non- 
equilibrium plasmas generated by nanosecond pulse discharges, and reviews of this work are given in $[28,29]$. Recent work on ignition by nanosecond discharges has included both experimental [30-32] and numerical [24, 30, 3234] studies of the effect of the plasma chemistry and electrodynamics. Also, recent advancements have been made on measuring and modeling ignition by transient plasma streamer discharge $[35,36]$. While significant progress has been made on modeling the fluid mechanics, chemistry, and plasma dynamics of spark discharge separately, full integration into a single numerical simulation has not been achieved. While the plasma chemistry and electrodynamics are beyond the scope of the current work and therefore not considered, they are critical for a complete model of the ignition process.

In the current work, high-speed schlieren visualization and two-dimensional simulations of spark discharge in a non-reactive gas and ignition are presented. The effect of electrode geometry on the flow field subsequent to the spark discharge and on ignition is investigated for three distinctly different electrode types: cylindrical with a $0.38 \mathrm{~mm}$ diameter, conical with a 6.35 base diameter, and blunt cylindrical electrodes with Teflon flanges. Only a few authors have considered conical electrodes [5, 10] and in these studies the base diameters of the electrodes were 1 to $2 \mathrm{~mm}$. Thin cylindrical electrodes have been considered by several authors $[4,5,7-10,15,16]$. In all the studies except $[4,15]$ the diameters of the cylindrical electrodes were 1 to $2 \mathrm{~mm}$; in the present study the diameter is $0.38 \mathrm{~mm}$, on the order of the thickness of the initial spark channel. Finally, in our work, flanged electrodes are also considered. This geometry is particularly important because flanged electrodes were used to obtain the classic minimum ignition energy and quenching distance data [37] that is still relied on extensively in the scientific literature and safety standards. The role of the flanges in the ignition process is not well understood and there have been few studies which consider flanged electrodes.

In both the simulations and experiments in the current study only very short sparks (on the order of $100 \mathrm{~ns}$ ) are considered. In some of the previous modeling work $[8,10,12]$ sparks with a breakdown phase followed by a long arc phase (10 to $100 \mu \mathrm{s}$ ) were used to simulate sparks from circuits with a significant inductance component, e.g., an automotive spark plug. Shorter duration $(<1 \mu \mathrm{s})$ sparks are more consistent with electrostatic discharge hazards in aviation and other industries. 


\section{Numerical Simulations}

\subsection{Model Description}

The spark discharge and ignition simulations were performed using direct numerical simulation (DNS) of the multicomponent, compressive, nonreactive and reactive Navier-Stokes equations. For the simulations of spark discharge in a non-reactive gas, the mass, momentum, and energy equations for two-dimensional, compressible, viscous, heat conducting flow are solved in cylindrical coordinates. The temperature dependence of the viscosity and thermal conductivity are described using the Sutherland law, and a power law model is used to account for the pressure dependence of the mass diffusivity [38]. For simulations of ignition, the gas is treated as a mixture of four perfect, reacting components and additional continuity equations for each of the chemical species are solved. The thermodynamic properties were evaluated as a function of temperature using the CHEMKIN-II library [39]. The thermodynamic model used in CHEMKIN is only valid up to 5000 Kelvin, and so constant properties are assumed for the high temperature phase. A detailed discussion of the numerical model and equations used is given in $[38,40]$.

In this first set of simulations the focus is on the fluid mechanics and not the chemistry, and so a highly simplified one-step chemistry model for a $15 \%$ hydrogen-air mixture was used to allow for higher resolution of the flow scales with a shorter computational time. The one-step chemistry model was developed in previous work $[3,41]$ to accurately simulate the characteristics of a one-dimensional hydrogen-air deflagration. By using this model, the effects of the complex chemical kinetics and the plasma chemistry are neglected and therefore must be taken into account in future improvements of the spark ignition simulations.

\subsection{Initial and Boundary Conditions}

In the present study, the primary objective is to investigate the influence of the electrode geometry on the fluid dynamics following the spark discharge and how the flow field affects the ignition process. Therefore, simulation of the spark breakdown phase and plasma formation is beyond the scope of the current work. Instead, a highly simplified model of the spark is used where it is approximated as a channel of gas at high temperature and pressure. This model neglects the important effects of the plasma chemistry as well as the highly complex electrodynamics and plasma formation process, which was 
shown in prior work [3] to significantly influence ignition. Future work to further develop and improve the spark ignition simulations would necessarily include more complex models for the spark.

The initial conditions are based on those used by Kravchik et al. [9] and Thiele et al. [10], which were motivated by the work of Maly and Vogel on spark discharges $[26,42]$. The initial conditions are those of the plasma channel at thermodynamic equilibrium approximately $60 \mathrm{~ns}$ after breakdown of the electrode gap as determined by Maly and Vogel [26, 42]. During the breakdown phase, as the electrical energy is transferred to the electrons and ions the gas temperature increases dramatically to tens of thousands of degrees Kelvin, causing the gas pressure to increase rapidly as well. This high temperature, high pressure channel of gas is approximately $40 \mu \mathrm{m}$ in diameter, corresponding to the diameter of the streamers that developed in the pre-breakdown phase. At approximately $60 \mathrm{~ns}$ after the onset of breakdown in a $1 \mathrm{~mm}$ electrode gap at 1 bar, Maly found that the gas temperature and pressure were approximately $35,000 \mathrm{~K}$ and $1 \mathrm{MPa}$, respectively [42]. Subsequent to breakdown, the electrical energy stored in the ignition circuit is deposited into the plasma channel. Sher and co-workers [43] found that an increase in the energy does not further increase the plasma temperature, but rather results in a larger diameter spark channel, and this energy deposition from the external circuit into the channel is sometimes considered as a constant volume process [43]. Therefore, in the current work the spark is modeled as a thin cylindrical channel between the electrodes at the post-breakdown conditions $(35,000 \mathrm{~K}$ and $1 \mathrm{MPa})$ with the channel diameter determined by the spark energy.

Assuming the spark energy is deposited under constant volume conditions, the volume of the spark channel is

$$
V_{c}=\frac{E_{\text {spark }}}{c_{V} \rho_{0}\left(T_{c}-T_{0}\right)}
$$

where $T_{c}$ is the temperature of the channel and $\rho_{0}$ and $T_{0}$ are the density and temperature of the ambient gas. Taking a cylindrical channel of length $L_{\text {gap }}$, then the channel radius is

$$
r_{c}=\left(\frac{V_{k}}{\pi L_{\text {gap }}}\right)^{1 / 2}
$$


In the spark discharge studies without ignition, the gas used in the simulations is air (79\% nitrogen, $21 \%$ oxygen) and the following values were assumed within the spark channel: $c_{V}=721 \mathrm{~J} / \mathrm{kg} \cdot \mathrm{K}, \rho_{0}=1.15 \mathrm{~kg} / \mathrm{m}^{3}, T_{k}=$ $35,000 \mathrm{~K}$; and outside the channel $T_{0}=300 \mathrm{~K}$ and $p_{0}=0.1 \mathrm{MPa}$. It should be noted again that the current work uses air and a one-step chemistry model for hydrogen combustion, and therefore neglects the plasma chemistry. In future work that includes a kinetics model for the plasma, the channel properties will have to be calculated as a function of temperature.

In both the experiments and simulations the spark energy used is $E_{\text {spark }}$ $=2 \mathrm{~mJ}$ and the spark gap is $L_{\text {gap }}=2 \mathrm{~mm}$. Using Equations 1 and 2, the volume and radius of the spark channel used for the initial condition is approximately $0.07 \mathrm{~mm}^{3}$ and $0.1 \mathrm{~mm}$, respectively. This estimate of the spark channel radius is consistent with experimental measurements [42]. The Ghost Fluid Method (GFM) is used to model the solid electrode boundary and enforce the no slip boundary condition, and the electrode is modeled as an adiabatic boundary so heat loss to the electrode is neglected. There are two planes of symmetry, the boundaries $r=0$ and $x=0$, so that only a quadrant of the flow is computed.

\subsection{Numerical Solution}

For the numerical solution of the equations described in Section 2.1, a hybrid WENO/centered-difference scheme developed by Ziegler and coworkers $[38,40]$ is used. To allow for highly-resolved simulations, this hybrid scheme is implemented into AMROC (Adaptive Mesh Refinement in Object-Oriented $\mathrm{C}++)$, a fluid-solver SAMR framework that uses an improved version of the block structured adaptive mesh refinement algorithm of Berger and Oliger [44] and Berger and Colella [45]. A short discussion of the key features of the solution method are provided in this section; complete details on the numerical method and are given in [38, 40].

The convective fluxes in the fluid are calculated with second order accuracy using a forward Euler integration method in combination with the MUSCUL finite volume scheme. Diffusion is modeled in the simulation using second order finite differences. The finite volume method solves for the convective fluxes, and then a diffusive flux is added before updating with forward Euler integration. The diffusive flux includes the viscous shear, heat conduction, mass diffusion, and diffusion of enthalpy by mass transport in the energy equation. A second-order, two-step Runge-Kutta method is used

to integrate the chemistry source terms. Finally, a Strang-splitting method 
is used for coupling of the fluid and chemistry time integration, allowing for second order time accuracy overall.

For the grid refinement, criteria are used that capture the physics of each length scale in the problem. The gradients of the density, radial and axial velocities, energy, and mass fractions are used for the convective, viscous, conductive, and flame thickness length scales, respectively. When a gradient across two cells becomes larger than a user-specified tolerance, a refinement level is added.

\section{Experiments}

Spark discharge and ignition experiments were performed in a 22 liter stainless steel combustion vessel. A low-energy, capacitive spark-discharge circuit was developed to generate short-duration sparks $(<1 \mu s)$ across the $2 \mathrm{~mm}$ spark gap. The spark current versus time is measured using a fast current transformer and is used to estimate to the energy deposited in the spark, which will be slightly lower than the energy stored in the capacitor $\left(1 / 2 C V^{2}\right)$. A detailed discussion of the spark ignition system and the spark energy estimation is given in [3].

A schlieren system was designed to visualize the spark gap region with high resolution by condensing the light from a 150 watt xenon arc lamp into a small circular field of view approximately $22 \mathrm{~mm}$ in diameter. A Vision Research Phantom v710 high-speed camera was used to take schlieren video at rates of 10,000 to 79,000 frames per second with resolutions from $800 \mathrm{x}$ 800 to $256 \times 256$, respectively. Windows on two parallel sides of the vessel allowed for visualization.

High-speed schlieren video was obtained of spark discharges in air using multiple camera speeds for three different electrode geometries. All the electrodes are made of tungsten to minimize surface corrosion during discharge. The cylindrical electrodes are made of tungsten wire with a $0.38 \mathrm{~mm}$ diameter. The conical electrodes have a base diameter of $6.35 \mathrm{~mm}$, a cone angle of 53 degrees, and the tips are rounded with a radius of curvature of approximately $0.8 \mathrm{~mm}$. The flanged electrodes have a cylindrical tungsten electrode with an approximate diameter of $1.6 \mathrm{~mm}$ surrounded by a round $19 \mathrm{~mm}$ diameter Teflon flange. Ignition tests in a 15\% hydrogen-air mixture were also performed at two camera speeds with each of the three electrode configurations. In all the tests the spark gap was fixed at $2 \mathrm{~mm}$. 


\section{Results}

\subsection{Spark Discharge in Non-Reactive Medium}

Images from high-speed schlieren visualization of a $2 \mathrm{~mJ}$ spark discharge in air using the 0.38 diameter cylindrical electrodes and images from the two-dimensional simulation at approximately the same time steps are shown in Figure 1. The video was taken at a rate of 79,069 frames per second with a total field of view of approximately $6.7 \mathrm{~mm}$ x $6.7 \mathrm{~mm}$. Dimensions are given on the images in millimeters, and the computational region is indicated on the first schlieren image by a white box. The first image is taken less than $12.6 \mu$ s after the spark breakdown and for discussion corresponds to time $t=0$. The spark breakdown creates a thin plasma channel at high temperature and pressure, as described in Section 2.2, and when the channel expands, a blast wave propagates outward while a rarefaction wave propagates inward toward the center of the channel. The rarefaction reflects at the center of the channel and propagates back outward and is seen trailing the blast wave in the pressure contours from the simulation. Initially, the shock wave is nearly a pure cylindrical wave except for very close to the electrode surface, where the wave is spherical in nature. Because the pressure gradient following a cylindrical shock wave is smaller than that following a spherical shock wave, the pressure is higher in the middle of the channel than next to the electrodes, causing gas to flow outward toward the electrode surface as illustrated in Figure 2. The flow separates and creates a clockwise-rotating vortex at the corner of the face and cylindrical body of the electrode, and additional vorticity is generated from the boundary layer due to the flow along the electrodes. The pressure gradient rapidly decreases and as the outward flow stops, the vortices propagate outward from the corner and pull fluid inward along the electrode as shown in both the schlieren visualization and simulation at approximately $10 \mu \mathrm{s}$.

The vorticity contours from the computation reveal that by $10 \mu \mathrm{s}$ a counter-rotating vortex pair has formed near the tip of the electrode, shown in Figure 3. The clockwise rotating vortex (top) is a result of the flow separation, and the counter-clockwise rotating vortex (bottom) is a result of the shear layer that develops due to flow moving outward against the inflow. The clockwise rotating vortex is convected towards the center of the channel by the inflow, and when the inflowing gas reaches the vertical plane of symmetry at approximately $40 \mu \mathrm{s}$ it turns vertical and convects the vortex up out of the channel until it is dissipated. Because there is a vertical plane of symmetry 

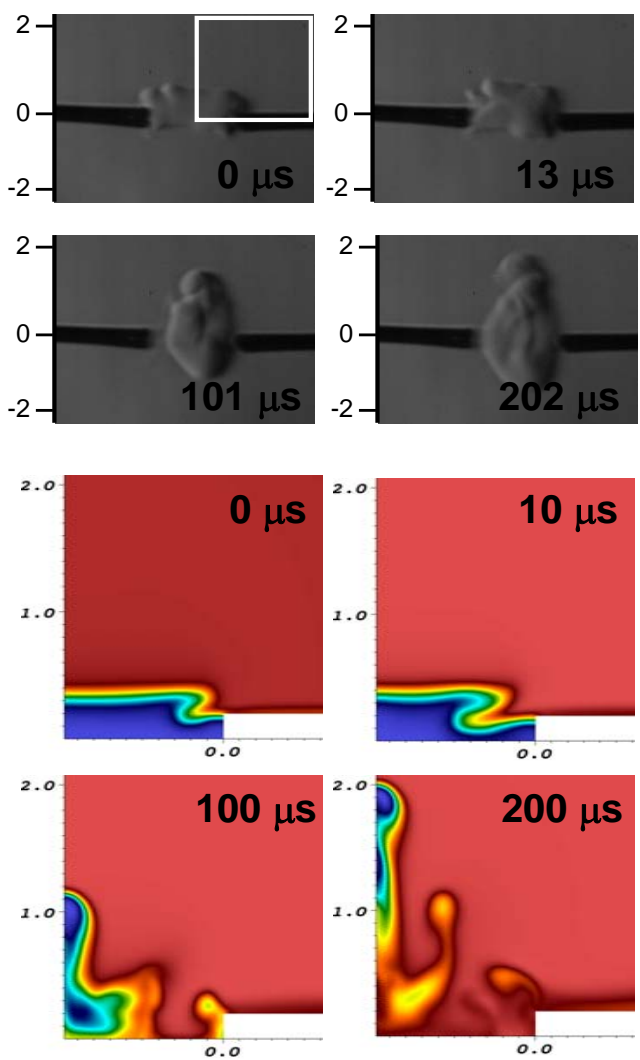

Figure 1: Images from high-speed schlieren visualization (upper set) and simulation (lower set) of a $2 \mathrm{~mJ}$ spark discharge in air with $0.38 \mathrm{~mm}$ diameter cylindrical electrodes. Distances shown are in $\mathrm{mm}$, simulation region corresponds to the quadrant outlined in white on the upper left schlieren image.

at the center of the channel, it is expected that a counter-clockwise rotating vortex would be generated from the other electrode and propagate upwards as part of a vortex pair. The schlieren visualization of the kernel clearly shows this phenomenon, as well as the symmetry about the $r=0$ plane. The temperature results from the simulation reveal that the vortex traps a kernel of hot gas, preventing it from being cooled by the gas inflow, and this hot kernel continues to propagate vertically from the center of the channel. The kernel cools quickly and its temperature decreases below $1000 \mathrm{~K}$ by 80 $\mu \mathrm{s}$. There is also a mixing region near the channel and the inflow of cool gas causes this region to be at significantly lower temperatures than the rising 


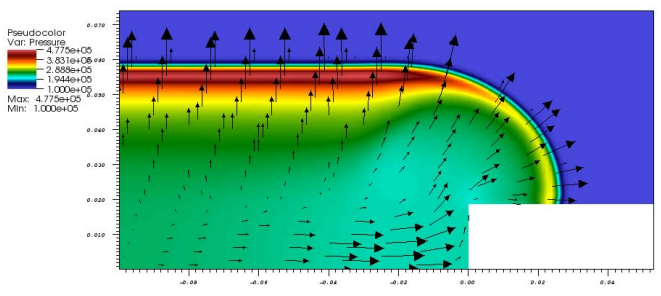

Figure 2: Simulated pressure field and velocity vectors showing the cylindrical and spherical portions of the blast wave at time $t=0.5 \mu \mathrm{s}$.

kernel. The major features of the flow field in the simulation, including the inflow of cold gas immediately following expansion of the spark channel, the rising hot kernel, and the mixing region are also observed in the schlieren visualization.

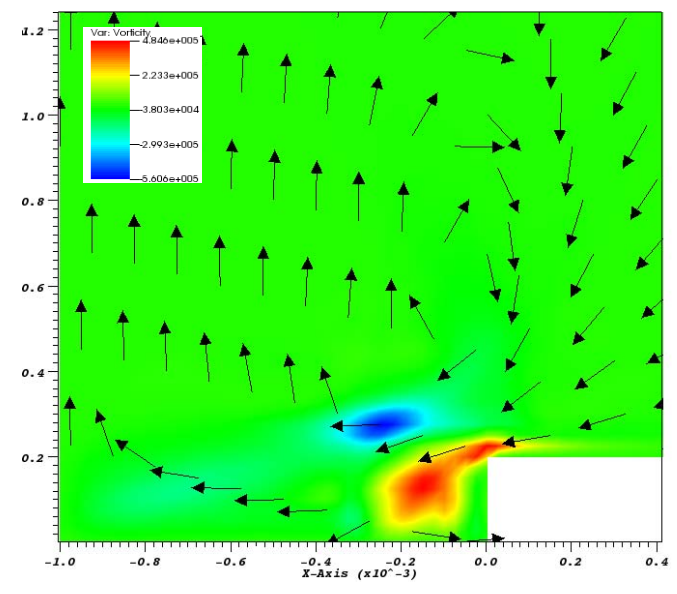

Figure 3: Simulation results (vorticity and velocity vectors) showing the vortex pair generated near the tip of the cylindrical electrode at time $t=10 \mu \mathrm{s}$.

Images from schlieren visualization of the spark discharge with the conical electrodes and the results from the two-dimensional simulation are shown in Figure 4. The images were taken at the same time steps as those for the cylindrical electrode case for comparison. In this geometry, the competition between spherical and cylindrical expansion is more predominant than in the cylindrical electrode case. Once again, a clockwise-rotating vortex is generated near the tip of the electrode due to flow separation and boundary 
layer vorticity and induces inflow into the channel. The vortex is weaker in this geometry than in the cylindrical geometry due to less flow separation, and as a result the inflow has a lower velocity. The vortex created by the flow separation is convected towards the center of the channel and then upward. Due to the lower rates of convection and entrainment of cold gas, the kernel cools slower than in the cylindrical electrode case, maintaining a temperature above $1000 \mathrm{~K}$ until $140 \mu \mathrm{s}$. The mixing region that forms near the gap is larger and at higher temperatures than in the cylindrical case. These flow features are also seen in the schlieren visualization, including the larger mixing region and slower propagation of the hot gas kernel. In comparison to the cylindrical electrodes, we have for the same energy a higher temperature gas kernel and larger mixing region, suggesting that for a given mixture, a lower spark energy would be needed for ignition.
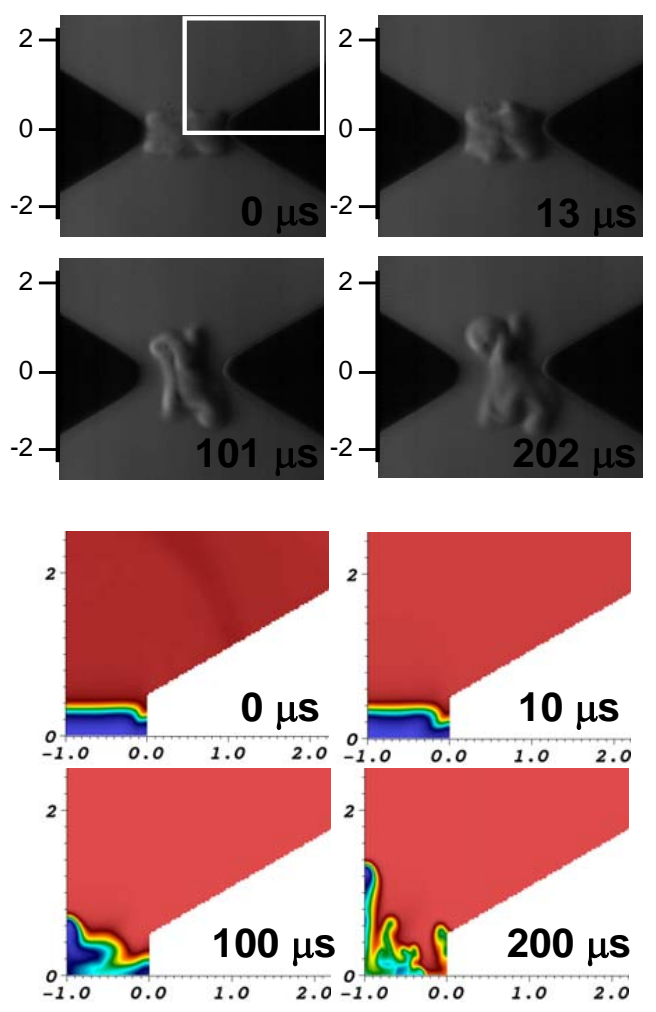

Figure 4: Images from high-speed schlieren visualization and simulation of a $2 \mathrm{~mJ}$ spark discharge in air with conical electrodes. 
The results of the schlieren visualization and computations for the third geometry, $1.6 \mathrm{~mm}$ diameter electrodes with Teflon flanges, are shown in Figure 5. In this geometry the expanding spark channel generates a purely cylindrical blast wave, and therefore there is no pressure gradient along the spark channel. However, in both the simulation and the schlieren visualization there is clearly inflow of gas towards the center of the channel caused by viscous effects. The vorticity field from the simulation shows that there is negative vorticity originating in the boundary layer at the right-hand flange and positive vorticity originating in the boundary layer at the left-hand flange. The vorticity diffuses into the flow to form a vortex pair which is clearly visible in the experiments. This weak vortex pair moves slowly outward, and the kernel is hotter for a longer time than in the other cases, maintaining a temperature above $1000 \mathrm{~K}$ until $340 \mu \mathrm{s}$. The confinement of the gas also results in a larger and hotter mixing region. Therefore, these results suggest that the lowest ignition energy would be required in this configuration and that the overall minimum ignition energy for a flammable gas is obtained using this geometry, as done by Lewis and von Elbe [37]. The generation of this vortex and subsequent hot kernel is a result not seen in previous simulations, and was captured by these simulations due to the high resolution and inclusion of viscous effects.

\subsection{Ignition of Hydrogen-Air Mixture}

Images from the schlieren visualization and images of the product (water in this case) from the simulation of ignition are shown in Figure 6 for the cylindrical electrode case. Both the experiment and computation show the inflow of cold reactant gas along the electrode which then rolls up with the hot product gas expanding rapidly outward to form a large vortex with the flame front on its surface. This part of the flame continues to burn outward, while the small rising kernel in the center of the channel forms the rest of the flame front.

Figure 7 shows images from the schlieren visualization and of the simulated product $\left(\mathrm{H}_{2} \mathrm{O}\right)$ for the ignition with the conical electrodes. The flame formation is very similar to the cylindrical electrode case as expected from the similarities in the fluid flow following spark discharge. Initially there is inflow along the electrode which forms a vortex with the outward flowing product gas. The flame front propagates outwards on the surfaces of this vortical structure and the rising gas kernel in the center of the channel. 

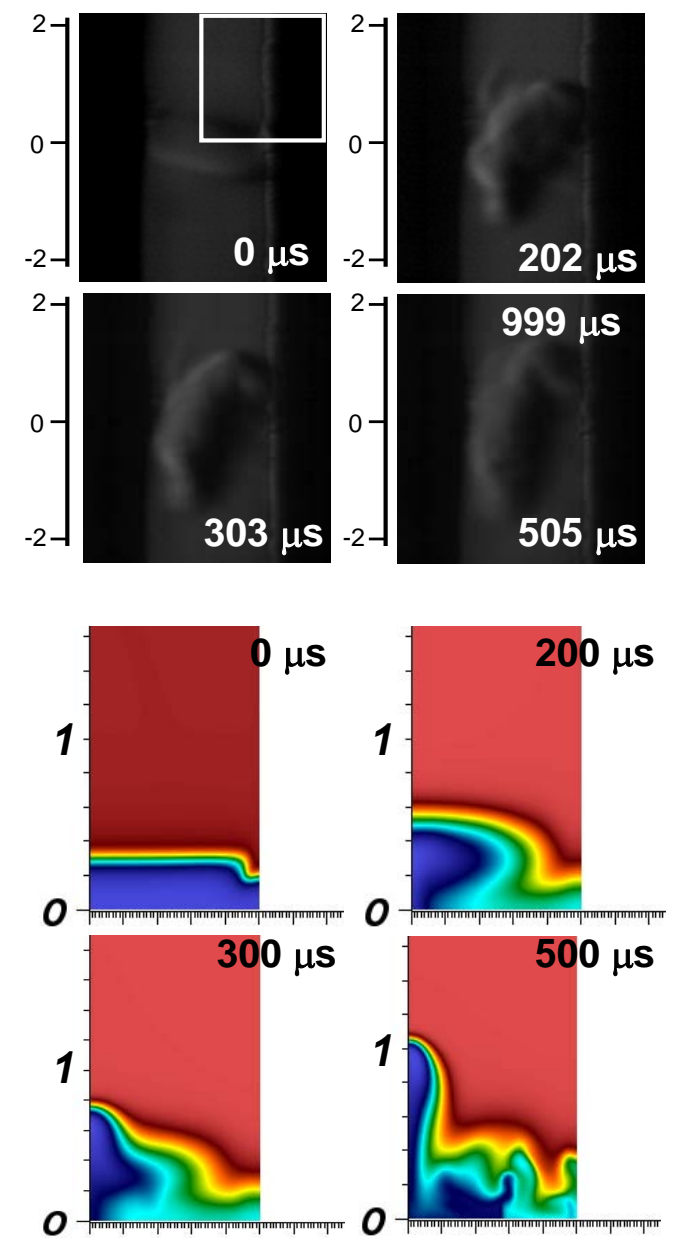

Figure 5: Images from high-speed schlieren visualization and simulation of a $2 \mathrm{~mJ}$ spark discharge in air with $1.6 \mathrm{~mm}$ diameter cylindrical electrodes with $19 \mathrm{~mm}$ diameter Teflon flanges.

Finally, the results for schlieren visualization and simulation of ignition with the flanged electrodes are shown in Figure 8. In the simulation, the flame front is curved due to the viscous flow velocity profile in the channel. The kernel shape is more pronounced in the schlieren images, and there is also some asymmetry of the flame front. The cause of this asymmetry is unclear, but three potential causes have been postulated. The first possible reason for the observed asymmetry of the flame front is an optical effect 

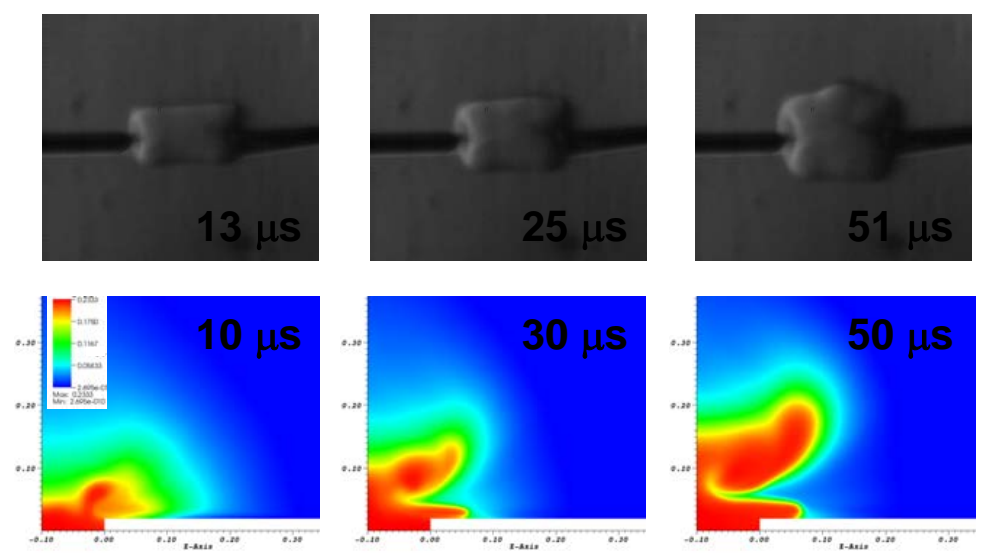

Figure 6: Images from high-speed schlieren visualization and simulation of ignition with the thin cylindrical electrodes.
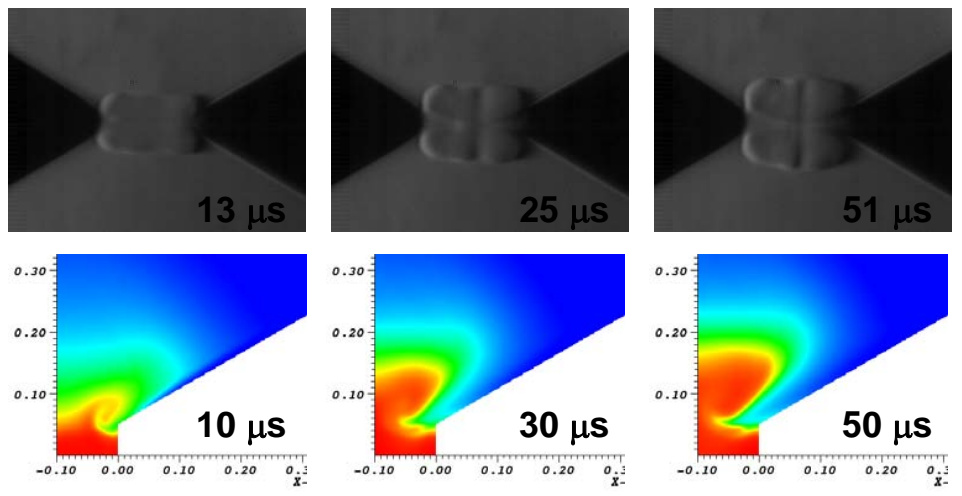

Figure 7: Images from high-speed schlieren visualization and simulation of ignition with the conical electrodes.

due to misalignment in the schlieren setup. Another possible cause is an actual fluid instability caused by the vortex pair being in close proximity. Finally, there may also be an asymmetry in the electrical discharge as there are different physical processes that dominate at the anode vs. the cathode. Further experiments and simulations would be needed to determine if the asymmetry is a physical phenomenon, e.g. a flow instability or cathode effect, or if it is caused by the optical system. Not shown in Figure 8 are the very interesting effects at late times, including the creation of a vortex pair at the 
outer edges of the flanges and the ingestion back into the flanged region due to the pressure becoming sub-atmospheric. This is only seen in the reacting simulations due to the higher velocities associated with the volume expansion of the burning gas. The propagating flame ultimately appeared to be very similar to observations of steady flames in 2-D channels [46].
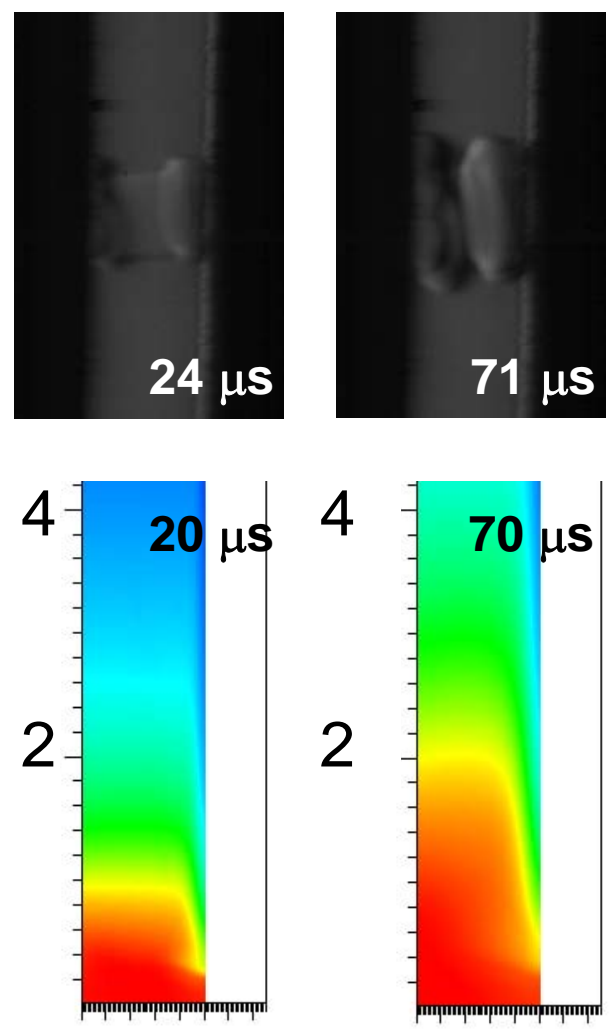

Figure 8: Images from high-speed schlieren visualization and simulation of ignition with the flanged electrodes.

\section{Conclusions}

High-speed schlieren visualization and numerical simulations were used to study the fluid mechanics following a spark discharge and the effect on the ignition process in a hydrogen-air mixture. The simulations were able to resolve the complex fluid mechanics processes and illustrated the important 
roles of the electrode geometry, pressure gradients, viscous effects, and flow confinement on the ignition process. By simulating both the compressible flow aspects at very early times as well as the later viscous and chemical reaction effects, the important features seen in the schlieren visualization were accurately reproduced by the simulations.

Initially, the flow field following a spark discharge is induced by the blast wave emitted from the high-temperature, high-pressure spark channel. The nature of the blast wave depends on the geometry, and consequently the details of the fluid mechanics of the evolving spark kernel will be greatly influenced by the electrode shape and spacing. The blast wave generates complex, unsteady pressure gradients that lead to simultaneous hot gas expansion in the gap and cold gas inflow along the electrode surfaces. Significant vorticity is generated in the shear layer and by the viscous effects at the boundaries, resulting in a hot gas kernel trapped by a vortex pair and a mixing region near the electrode tips. The temperature and rate of cooling of the hot kernel and mixing region are significantly influenced by the electrode geometry and will have a critical effect on the chemistry and flame formation.

In the flanged electrode geometry, it was expected that purely cylindrical expansion would be seen. However, both the simulations and experiments showed that even in this two-dimensional geometry, the viscous effects lead to multidimensional flow, a result not shown in previous modeling work. Therefore, including viscosity in simulations of ignition is extremely important as it has a large effect on the flow field in the flanged electrode case as well as in the other two geometries. In the flanged geometry, the viscous effects generate vortices that trap a hot gas kernel similar to that seen with the other two electrode geometries. The high degree of confinement by the flanges results in a hotter gas kernel and mixing region, which suggests that the lowest minimum ignition energy and ignition delay time would be obtained using flanged electrodes. Similarly, the largest ignition energy may be required for the thin cylindrical electrode case due to the high rate of gas cooling.

Schlieren visualization and two-dimensional simulations of ignition in a $15 \%$ hydrogen-air mixture were also conducted to study the effect of the flow field generated by the spark discharge. The flame formation process was comparable in the cylindrical and conical electrode cases due to the similarities of the flow fields in the two geometries. The flame front is first initiated on the boundary of the vortex generated by the boundary layer effects due to the efficient mixing. The remainder of the flame front develops on the outside of the hot gas kernel trapped by the second vortex. The 
flame formation process is significantly influenced by viscosity in the flanged electrode case, with the boundary layer velocity profile producing a curved flame front.

In the current study, a highly simplified model for the spark was used and only one set of initial conditions for the spark channel was studied. The simplifications employed in the spark model and the initial conditions will both influence the simulation results. A parametric study is needed to characterize this influence, which is outside the scope of the current work; however, some general conclusions can be postulated. A major simplification used in the spark model is the use of air or one-step hydrogen chemistry, which neglects the complex plasma chemistry. The generation of radicals in the plasma and the plasma reactions will affect the chemistry, possibly accelerating the reaction rates and promoting ignition at lower spark energies. The inclusion of plasma chemistry will also affect the initial temperature and size of the spark channel. The initial conditions of the spark channel will also be influenced by the overall spark energy and the temporal energy addition. In the current work, a spark energy of $2 \mathrm{~mJ}$ deposited instantaneously in a constant volume process was used in all cases; in future work, the energy deposition should be varied and modeled as a transient process. Ziegler [38] began this work by varying the spark channel diameter in both one and two-dimensional axisymmetric simulations and observing if a self-sustained deflagration is established. While the current simulations use simplified spark models and initial conditions, the favorable comparison to the experimental results provides confidence in the overall conclusions reached on the sparkinduced fluid dynamics and the effect on ignition.

The results of this work provide new insights on the roles of the various physical phenomena in spark kernel formation and ignition. In particular, the simulations and experiments demonstrated the important role of viscosity, the effects of vorticity induced by pressure gradients and the boundary layer, the influence of the electrode geometry, and the effect of hot gas confinement. Therefore, we conclude that accurate resolution of the scales involved in the fluid dynamics, pressure gradients, and viscous processes is critical in developing a realistic model of spark ignition.

\section{Acknowledgments}

Sally Bane was supported by The Boeing Company through a Strategic Research and Development Relationship Agreement CT-BA-GTA-1. Jack 
Ziegler was supported by the Department of Energy Computational Science Graduate Fellowship program (DOE CSGF).

\section{References}

[1] S. P. M. Bane, J. L. Ziegler, P. A. Boettcher, S. A. Coronel, J. E. Shepherd, Journal of Loss Prevention in the Process Industries 26 (2013) 290-294.

[2] S. A. Coronel, R. Mevel, S. P. M. Bane, J. E. Shepherd, Proceedings of the Combustion Institute 34 (2013) 895-902.

[3] S. P. M. Bane, Spark Ignition: Experimental and Numerical Investigation with Application to Aviation Safety, Ph.D. thesis, California Institute of Technology, 2010.

[4] M. Kono, K. Niu, T. Tsukamoto, Y. Ujiie, Proceedings of the Combustion Institute 22 (1988) 1643-1649.

[5] M. Akram, AIAA Journal 34 (1996) 1835-1842.

[6] R. Reinmann, M. Akram, Journal of Physics D - Applied Physics 30 (1997) 1125-1134.

[7] O. Ekici, O. A. Ezekoye, M. J. Hall, R. D. Matthews, Journal of Fluids Engineering 129 (2007) 55-65.

[8] K. Ishii, T. Tsukamoto, Y. Ujiie, M. Kono, Combustion and Flame 91 (1992) 153-164.

[9] T. Kravchik, E. Sher, J. B. Heywood, Combustion Science and Technology 108 (1995) 1-30.

[10] M. Thiele, J. Warnatz, U. Maas, Combustion Theory and Modeling 4 (2000) 413-434.

[11] M. Thiele, S. Selle, U. R. J. Warnatz, U. Maas, Proceedings of the Combustion Institute 28 (2000) 1177-1185.

[12] M. Thiele, J. Warnatz, A. Dreizler, S. Lindenmaier, R. Schießl, U. Maas, A. Grant, P. Ewart, Combustion and Flame 128 (2002) 74-87. 
[13] T. Yuasa, S. Kadota, M. Tsue, M. Kono, H. Nomura, Y. Ujiee, Proceedings of the Combustion Institute 29 (2002) 743-750.

[14] J. Han, H. Yamashita, N. Hayashi, Combustion and Flame 157 (2010) $1414-1421$.

[15] J. Han, H. Yamashita, N. Hayashi, International Journal of Hydrogen Energy 36 (2011) 9286-9297.

[16] S. Nakaya, K. Hatori, M. Tsue, M. Kono, D. Segawa, T. Kadota, Journal of Propulsion and Power 27 (2011) 363-370.

[17] H. L. Olsen, R. B. Edmonson, E. L. Gayhart, Journal of Applied Physics 23 (1952) 1157-1162.

[18] D. Bradley, I. L. Critchley, Combustion and Flame 22 (1974) 143-152.

[19] M. Champion, B. Deshaies, G. Joulin, K. Kinoshita, Combustion and Flame 65 (1986) 319-337.

[20] P. L. Pitt, R. M. Clements, D. R. Topham, Combustion Science and Technology 78 (1991) 289-314.

[21] S. Au, R. Haley, P. Smy, Combustion and Flame 88 (1992) 50-60.

[22] V. S. Arpaci, Y. Ko, M. T. Lim, H. S. Lee, Combustion and Flame 135 (2003) 315-322.

[23] R. Ono, N. Masaharu, S. Fujiwara, S. Horiguchi, T. Oda, Journal of Applied Physics 97 (2005) 123307.

[24] K. Eisazadeh-Far, F. Parsinejad, H. Metghalchi, J. C. Keck, Combustion and Flame 157 (2010) 2211-2221.

[25] M.-W. Peng, S. Shy, Y.-W. Shiu, C.-C. Liu, Combustion and Flame 160 (2013) 1755-1766.

[26] R. Maly, M. Vogel, Proceedings of the Combustion Institute 17 (1979) $821-831$.

[27] R. Ono, T. Oda, Combustion and Flame 152 (2008) 69-79. 
[28] I. V. Adamovich, I. Choi, N. Jiang, J. H. Kim, S. Keshav, W. R. Lempert, E. Mintusov, M. Nishihara, M. Samimy, M. Uddi, Plasma Sources Science and Technology 18 (2009) 034018.

[29] A. Starikovskiy, N. Aleksandrov, Progress in Energy and Combustion Science 39 (2013) 61-110.

[30] N. L. Aleksandrov, S. V. Kindysheva, I. N. Kosarev, S. M. Starikovskaia, A. Y. Starikovskii, Proceedings of the Combustion Institute 32 (2009) 205-212.

[31] Z. Yin, I. V. Adamovich, W. R. Lempert, Proceedings of the Combustion Institute 34 (2013) 3249-3258.

[32] M. A. Deminskii, I. V. Chernysheva, S. Y. Umanskii, M. I. Strelkova, A. E. Baranov, I. V. Kochetov, A. P. Napartovich, T. Sommerer, S. Saddough, J. Herbon, B. V. Potapkin, Russian Journal of Physical Chemistry B 7 (2013) 410-423.

[33] N. L. Aleksandrov, S. V. Kindysheva, E. N. Kukaev, S. M. Starikovskaya, A. Y. Starikovskii, Plasma Physics Reports 35 (2009) 867-882.

[34] J. Poggie, I. Adamovich, N. Bisek, M. Nishihara, Plasma Sources Science and Technology 22 (2013) 015001.

[35] T. Langer, D. Markus, U. Maas, in: 42nd AIAA Plasmadynamics and Lasers Conference. AIAA 2011-3450.

[36] D. R. Singleton, M. A. Gundersen, IEEE Transactions on Plasma Science 39 (2011) 2214-2215.

[37] B. Lewis, G. von Elbe, Combustion, Flames and Explosions of Gases, Academic Press, New York, 1961.

[38] J. L. Ziegler, Simulations of Compressible, Diffusive, Reactive Flows with Detailed Chemistry Using a High-Order Hybrid WENO-CD Scheme, Ph.D. thesis, California Institute of Technology, 2012.

[39] R. J. Kee, F. M. Rupley, J. A. Miller, Chemkin-II: A Fortran Chemical Kinetics Package for the Analysis of Gas-Phase Chemical Kinetics, Technical Report SAND-89-8009, Sandia National Laboratories, Livermore, California, 1989. 
[40] J. L. Ziegler, R. Deiterding, J. E. Shepherd, D. I. Pullin, Journal of Computational Physics 230 (2011) 7598-7630.

[41] S. P. M. Bane, J. L. Ziegler, J. E. Shepherd, Development of One-Step Chemistry Models for Hydrogen-Air and Propane-Air Systems, Technical Report FM2010-002, Graduate Aeronautical Laboratories, California Institute of Technology, 2010.

[42] R. Maly, Fuel Economy in Road Vehicles Powered by Spark Ignition Engines, Springer, pp. 91-148.

[43] E. Sher, J. Ben-ya'ish, T. Kravchik, Combustion and Flame 89 (1992) 186-194.

[44] M. Berger, J. Oliger, Journal of Computational Physics 53 (1984) 484512.

[45] M. Berger, P. Colella, Journal of Computational Physics 82 (1988) 6484.

[46] J. Jarosinski, Combustion Phenomena: Selected Mechanisms of Flame Formation, Propagation, and Extinction, CRC Press, pp. 15-25. 